\title{
Editorial: Global Food and Nutrition Security Under Changing Climates
}

\author{
Waqar Ahmad ${ }^{1,2 *}$, Najeeb Ullah ${ }^{3,4 *}$, Ling X $\mathbf{u}^{5}$ and Ayman El Sabagh ${ }^{6,7}$ \\ ${ }^{1}$ School of Agriculture and Food Sciences, The University of Queensland, St Lucia, QLD, Australia, ${ }^{2}$ National Centre of \\ Excellence in Geology, University of Peshawar, Peshawar, Pakistan, ${ }^{3}$ Queensland Alliance for Agriculture and Food \\ Innovation, Centre for Plant Science, The University of Queensland, St Lucia, QLD, Australia, ${ }^{4}$ Faculty of Science, Universiti \\ Brunei Darussalam, Bandar Seri Begawan, Brunei, ${ }^{5}$ Zhejiang Province Key Laboratory of Plant Secondary Metabolism and \\ Regulation, College of Life Sciences and Medicine, Zhejiang Sci-Tech University, Hangzhou, China, ${ }^{6}$ Department of Field \\ Crops, Faculty of Agriculture, Siirt University, Siirt, Turkey, ${ }^{7}$ Department of Agronomy, Faculty of Agriculture, Kafrelsheikh \\ University, Kafrelsheikh, Egypt
}

Keywords: food security, climate change, micronutrient quality of food, sustainable yields, farm-level strategies, sustainable future

\section{Editorial on the Research Topic}

\section{Global Food and Nutrition Security Under Changing Climates}

Global climatic change poses a serious threat to crop production and food security. For instance, unpredictable weather events affecting the frequency of rains, floods, droughts, and extreme heat have already impacted global output and quality of staple crops and, thus, food and nutrition security (Ray et al., 2015). It has been estimated that climatic variability causes $\sim 1.6$ and 5.0 million tons (MT) annual reduction in global rice and wheat yields, respectively (Ray et al., 2015; Arora, 2019). This translates into a considerable loss of consumable calories and nutrient and adds to the challenge of feeding the growing world population ( $~ 10$ billion in 2050).

This editorial draws on articles published on this important Research Topic. The research findings highlight the significance of genotype and environmental interactions in determining how climate interacts with crop productivity. Crop plants show physiological modifications to respond to climatic stresses, although these modifications may impact yield and nutritional quality. However, innovative techniques that integrate genetic and management strategies for crop adaptation in the changing environments are essential to ensure global food security (Henry, 2020). Plant growth regulators (PGRs) offer an opportunity to induce stress tolerance and resilience of production system to changing climate. Indeed, PGRs have successfully been used in the cropping system to mitigate stress-induced changes in physiological functioning of crops (Najeeb et al., 2015; EL Sabagh et al.). Despite significant yield advancement achieved through improved management/breeding techniques, sustaining nutritional quality of the produce has been quite challenging, particularly for crop producers in developing countries. However, recent progress in the field of crop nutrition management through soil and plant agronomy could help to meet this challenge. For example, improved nutritional quality and wheat grain yield increases have been achieved through Zn fertilizer application, including in Pakistan (reviewed by Joy et al., 2017). Similarly, in an ex-ante analysis (10-yr data and associated parameters) of maize-based cropping systems, Manzeke-Kangara et al. (2021) reported that soil fertility management interventions with mineral NPK, organic nutrient inputs, and Zn fertilizers could increase dietary $\mathrm{Zn}$ supply in Zimbabwe and promote its cycling in cropping systems.

Conservation of soil resources is an essential foundation for health because most terrestrial biodiversity is found within the soil, and $98.8 \%$ of the daily calories consumed by humans $(2,849$ kcal per capita) come from soil with only $1.2 \%$ (35 kcal per capita) from aquatic sources (FAO, 2018). While the role of soil nutrients in crop production has been well-recognized in agricultural 
research and practice, the importance of soil and sustainable soil management interventions for human health has received relatively little attention. Fan et al. (2008) reported declining concentration of mineral elements in wheat grown in the UK, coinciding with the introduction of high-yielding semidwarf varieties. Declines in nutritional content of crops may be compounded in areas where agricultural soils have been degraded due to intensive farming and conventional crop management. Micronutrient deficiencies in soil-plant systems and in humans are increasingly well-documented, particularly in low- and middle-income countries (Ahmad et al., 2012; Lowe et al., 2018; Ishfaq et al., 2021).

Deficiency of micronutrients in staple foods is one of the causes of malnutrition and it affects a large population in the developing world. Approximately 2 billion people suffer from $\mathrm{Fe}$ and $\mathrm{Zn}$ deficiency globally, with greater prevalence in regions with predominantly cereal-based diets (Gregory et al., 2017). Severity of malnutrition of these micronutrients has been linked with their low intake through staple food crops (Ishfaq et al., 2021), and the concentration of mineral micronutrients in staple cereals varies spatially due to soil and landscape factors (Gashu et al., 2021). Globally, nearly 7.3\% (49.5 M) children are atrophied/wasted (low weight-for-height), 21.9\% (149 M) stunted (low height-for-age), and 5.9\% (40.1 M) overweight because of multi-faceted malnutrition (UNICEF, 2020).

Deficiency of elements such as $\mathrm{Zn}(\mathrm{ZnD})$ and iron $(\mathrm{FeD})$ negatively affects human health and is a serious public health nutrition concern. Globally, $17.3 \%$ of the population is at risk of $\mathrm{Zn}$ deficiency due to inadequate dietary intake with up to $30 \%$ of vulnerable populations in some parts of the world (Wessells and Brown, 2012). Similarly, FeD is one of the most common nutrient deficiencies globally, affecting $>25 \%$ of the population. The functions of $\mathrm{Zn}$ include but are not limited to helping people resist infectious diseases and healthy pregnancies (Ahmad et al., 2012). Good soil management could potentially reduce the overall deficiency of dietary nutrients. A negative correlation between grain yield and quality is often seen due to the "nutrient dilution" effect (Jarrell and Beverly, 1981; Simmonds, 1995); current global food systems are setup to prioritize increasing grain yields, with a lesser interest in nutritional quality improvement. Hence, increasing food production alone does not resolve the malnutrition problem, which is affected more by consumption patterns of calories and nutrients than by lack of food availability. On a global scale, $\sim 1$ billion people consume too few calories, $\sim 3$ billion have insufficient micronutrients, and $>2.5$ billion consume too much. These three burdens, which often co-exist in the same community and even in the same household, comprise the central challenge to achieving food and nutrition security for all (Godfray and Garnett, 2014).

Breeding efforts predominantly focus on increasing crop yields under the changing climates. Key physiological processes associated with grain yield production, especially carbon assimilation and sink-source relations are highly sensitive to environmental variations (Ullah and Chenu, 2019). While these processes also determine grain quality, climatic variability is likely to affect the nutritional quality of other crop groups (Alae-Carew et al., 2020). Climatic extremes further exacerbate the uncertainty of water and nutrient availability for future food production. However, the adoption of any innovative technology, including willingness to use PGRs and/or subsoil reclamation by farmers (growers), is driven by multiple factors that influence decision-making to implement specific techniques. Such determining factors include limitations to technical knowledge and environmental awareness, economic and farm-level considerations, and growers' beliefs and risk perception (Hinzmann et al.).

Family farms are critical actors in achieving the sustainable development goals (SDGs). Related SDGs include, 1 (eradicating poverty), 2 (achieving zero hunger), 10 (addressing inequalities), and 12 (achieving more sustainable production patterns). According to the latest estimates, out of total $608 \mathrm{M}$ farms, $90 \%$ are family farms that constitute $70-80 \%$ of the global farmland and produce $\sim 80 \%$ of the world food in value terms. The small farms ( $<2$ hectares, ha) account for $84 \%$ of all farms worldwide and produce $\sim 36 \%$ of the food. Farms in the range of $2-5$ ha, account for $10 \%$ of all farms and control $6 \%$ of the land. The largest $1 \%$ of farms in the world (>50 ha) operate $\sim 70 \%$ of the world's farmland (Lowder et al., 2019). We can categorize these farms associated to the small-scale, medium-scale, and large-scale growers. We propose here, strategies for all scales of growers both in developing and developed economies for micronutrient quality of food without compromising yields.

Currently operational farming systems can be broadly categorized into distinct groups based on farm size, access to resources (i.e., machinery, capital, innovative techniques) and level of linkages with larger economic systems. At the farm level, sustainable production is a goal of farming business, which is regulated by price volatility and economic inputs. To make agricultural systems more resilient to changing climate and economy, innovative techniques should be introduced. Adoption of these practices particularly by the small-scale farmers, will contribute to improving agricultural income and reducing poverty, thereby increasing food and nutritional security. To achieve this, a successful farm entrepreneurship requires extra support from extension agents for scaling-out and facilitating linkages along the value chain. This approach can help develop public policies and investments to support family farming, to increase the productivity of smallholders and improve rural livelihoods (as endorsed by the UN Decade of Family Farming 2019-28).

Out of the suitable, cost-effective strategies, biofortification is the one available for resource-poor farmers. Crop biofortification is the achievement of greater concentrations of micronutrients in the edible portion of crops, through selective breeding of micronutrient(s)-rich existing lines and varieties and/or the application of micronutrient fertilizers. Biofortified varieties for cereal crops have been released in many developing countries such as India, Pakistan, Bangladesh, Indonesia and Nepal, and many countries in Latin America/Caribbean and Africa (HarvestPLUS https://www.harvestplus.org). Biofortification of staple crops has emerged as a win-win approach to combat the rising malnutrition concerns. The rice-wheat cropping system is the world's most extensive agricultural production system ( $\sim 16 \mathrm{M}$ ha in India, Nepal, Pakistan, and Bangladesh, 
and $\sim 85 \%$ of this area falls in Indo-Gangetic plains that is among the world's most densely populated areas with a total population $\sim 480 \mathrm{M}$ ); it would be prudent to target this cropping system to combat malnutrition. Cost-effectiveness and feasibility considerations affect the adoption of new technologies, with additional challenges arising during the COVID-19 pandemic. Technologies that required only slight variations/adjustments in farmers' practices without affecting their current level of investment are likely to be most effective. For the largescale/progressive growers, other management plans could be worth-considering for an increased return on investment besides valuation/gain in quality and quantity parameters.

\section{RECOMMENDATION}

\section{Policy Interventions}

- One health policy should be considered for improving the state of food and nutritional security. The policy frameworks should address both surface and sub-surface constraints and exploit above-ground and below-ground resources for crop production.

- Enhance the research capacity of relevant organizations to make reliable predictions of climatic change, impact assessment on food production, nutrient flows and to develop appropriate adaptation and resilience measures.

- Develop capacity of farming community to take advantage of the scientific findings of relevant research organizations.

- More research projects/funds to be allocated for assessment studies, development projects for new crop varieties with increased resilience to changing climate and sustaining density of micronutrients and/or micronutrients' loading.

- Fertilizer companies and other similar businesses be stimulated to endow $5 \%$ or more of their net revenue on research, innovation, and commercialization. Measures should be taken to develop and implement sustainable approaches for small, medium, and progressive growers.

- Steps also should be taken to prepare remote sensing databased nutrition-sensitive hubs across the dominant cropping zones at global, regional, and country-scales.

- Access to road and rail infrastructure should be strengthened (with special consideration of small- and medium-scale farmers) for better connectivity between urban and rural areas in order to minimize food losses during transportation from field to market and return of wastes to the land for a "circular economy."

\section{Research and Development Initiatives}

- Further R\&D is warranted to assess processes specifically affecting the translocation of micronutrients from soil to grain, so that the biofortification approach does not over-rely on additional foliar sprays of target micronutrients which may not be feasible in many contexts.

- Fertilizer recommendations in many parts of the developing world are conventional and must be updated with researchbased evidence considering specific environmental factors and the changing climate.
- The production technologies of various important crops also need to be revisited as per the respective cropping zone(s) to make these adaptable to the climate change scenarios.

- 4Rs of nutrient stewardship - the Right source of nutrient, applied at the Right rate and Right time, and in the Right place-with sustainable soil management practices are known to increase productivity, bolster soil health and improve crop quality, particularly for macronutrients/fertilizers. $4 \mathrm{R}$ packages for the micronutrients application with aim to increase both yield and quality of the produce are much needed.

- Studies on cropping patterns and production technologies need to be updated considering the climate change for improved productivity.

- Micronutrient use scenarios should be simulated for cerealbased food production regions under different moisture and temperature regimes. Inputs and outputs must be valued in terms of both potential yield gains as well as health gains in the population. Such studies will explore the relationship between soil fertility and human health, and outline strategies to ameliorate micronutrient deficiencies in the food chain.

- Nutrient efficient genotypes should be developed using molecular tools (e.g., CRISPR-Cas9) to improve nutrient(s) use efficiencies at global scale.

- Scientific knowledge and related technical skills should be shared and promoted across the globe, including trainings on the transfer of laboratory scale analytical skills.

\section{Outreach Campaigns}

- There is a need to document indigenous best management practices to increase nutrient density in cereal grains (for example $\mathrm{Zn}$ and Fe and other minerals).

- Development of technical brochures related to micronutrients and other minerals should be done in local languages and placed at official websites of Agriculture Extension Department(s) which could further be considered for promotion via social, digital, radio, and print media.

- Agriculture awareness program (at least half hour a day) should be initiated at mainstream media channels, as currently no such program is available.

- Role of micronutrients and fertilizers be made part of national curriculum with emphasis about their use to enhance efficiency and improve nutrition management knowledge of farmers. This would also help raise awareness among the future farmers about role of fertilizers in food security.

- Popular fertilizers within a specific country be considered for coating with the micronutrients and minerals that are widely deficient.

- Fertilizer industry should be made part of any such media campaign so that program effectiveness could be enhanced and scaled.

- Civil society should be taken on board for any of the awareness campaigns that are run with an aim to improve food security.

- International and regional agencies/consortia such as Asian Soil Partnership, European Soil Partnership, African Soil Partnership (Initiative of Global Soil Partnership, 
UN-FAO), Scaling-Up Nutrition (SUN), Nutrition International, Global Alliance for Improved Nutrition (GAIN), and Society for Environmental Geochemistry and Health (SEGH), should collaborate to ensure supply of $\mathrm{Zn} / \mathrm{Fe}$ biofortified varieties along with the technology packages specifically for small, medium, and progressive growers. The consortium partners must work in collaboration to design research and incentive-based policy frameworks.

\section{AUTHOR CONTRIBUTIONS}

WA drafted the story line of the editorial. NU, LX, and AE refined the story line and contributed references and insights to the

\section{REFERENCES}

Ahmad, W., Watts, M. J., Imtiaz, M., Ahmed, I., and Zia, M. H. (2012). Zinc deficiency in soils, crops and humans. A review. Agrochimica 2, 65-97. doi: $10.5772 / 36702$

Alae-Carew, C., Nicoleau, S., Bird, F. A., Hawkins, P., Tuomisto, H. L., Haines, A., et al. (2020). The impact of environmental changes on the yield and nutritional quality of fruits, nuts and seeds: a systematic review. Environ. Res. Lett. 15:023002. doi: 10.1088/1748-9326/ab5cc0

Arora, N. K. (2019). Impact of climate change on agriculture production and its sustainable solutions. Environ. Sustain. 2, 95-96. doi: 10.1007/s42398-019-00078-w

Fan, M. S., Zhao, F. J., Fairweather-Tait, S. J., Poulton, P. R., Dunham, S. J., and McGrath, S. P. (2008). Evidence of decreasing mineral density in wheat grain over the last 160 years. J. Trace Elem. Med. Biol. 22, 315-324. doi: 10.1016/j.jtemb.2008.07.002

FAO (2018). FAOSTAT. Food and Agriculture Organization of the United Nations, Statistics Division. Available online at: http://www.fao.org/faostat/en/\#data/QC (accessed December 12, 2021).

Gashu, D., Nalivata, P. C., Amede, T., Ander, E. L., Bailey, E. H., Botoman, L., et al. (2021). The nutritional quality of cereals varies geospatially in Ethiopia and Malawi. Nature 594, 71-76. doi: 10.1038/s41586-021-03559-3

Godfray, H. C. J., and Garnett, T. (2014). Food security and sustainable intensification. Philos. Trans. R. Soc. Lond. B. Biol. Sci. 369:20120273. doi: 10.1098/rstb.2012.0273

Gregory, P. J., Wahbi, A., Adu-Gyamfi, J., Heiling, M., Gruber, R., Joy, E. J., et al. (2017). Approaches to reduce zinc and iron deficits in food systems. Global Food Secur. 15, 1-10. doi: 10.1016/j.gfs.2017.03.003

Henry, R. J. (2020). Innovations in plant genetics adapting agriculture to climate change. Curr. Opin. Plant Biol. 56, 168-173. doi: 10.1016/j.pbi.2019.11.004

Ishfaq, M., Wakeel, A., Shahzad, M. N., Kiran, A., and Li, X. (2021). Severity of zinc and iron malnutrition linked to low intake through a staple crop: a case study in east-central Pakistan. Environ. Geochem. Health 43, 4219-4233. doi: 10.1007/s10653-021-00912-3

Jarrell, W. M., and Beverly, R. B. (1981). The dilution effect in plant nutrition studies. Adv. Agron. 34, 197-224. doi: 10.1016/S0065-2113(08)60887-1

Joy, E. J. M., Ahmad, W., Zia, M. H., Kumssa, D. B., Young, S. D., Ander, E. L., et al. (2017). Valuing increased zinc (Zn) fertilizer use in Pakistan. Plant Soil. 411, 139-150. doi: 10.1007/s11104-016-2961-7

Lowder, S. K., Sánchez, M. V., and Bertini, R. (2019). Farms, Family Farms, Farmland Distribution and Farm Labour: What Do We Know? FAO Agricultural Development Economics Working Paper 19-08. FAO, Rome.

Lowe, N. M., Khan, M. J., Broadley, M. R., Zia, M. H., McArdle, H. J., Joy, E. J., et al. (2018). Examining the effectiveness of consuming flour made from agronomically biofortified wheat (Zincol-2016/NR-421) for improving $\mathrm{Zn}$ status in women in a low-resource setting in Pakistan: study protocol for impact of the papers included in this Research Topic. All authors contributed to the article and approved the submitted version.

\section{ACKNOWLEDGMENTS}

We thank all the authors who submitted their work to the Research Topics, the support of professional editorial staff at Frontiers, and the invaluable help of reviewers in manuscript evaluation. WA acknowledges Munir Zia (Coordinator Biofortification with Zinc and Iron for Eliminating Deficiency (BIZIFED) Project Pakistan Chapter) to help condense various recommendations. We are thankful to Edward Joy (London School of Hygiene and Tropical Medicine, University of London) for thought-provoking discussions on how to scale up nutrition across different regions of the world.

a randomised, double-blind, controlled cross-over trial (BiZiFED). Brit. Med. J. Open 8:e021364. doi: 10.1136/bmjopen-2017-021364

Manzeke-Kangara, M. G., Joy, E. J. M., Mtambanengwe, F., Chopera, P., Watts, M. J., Broadley, M. R., et al. (2021). Good soil management can reduce dietary zinc deficiency in Zimbabwe. CABI Agric. Biosci. 2, 1-12. doi: 10.1186/s43170-021-00057-4

Najeeb, U., Atwell, B. J., Bange, M. P., and Tan, D. K. (2015). Aminoethoxyvinylglycine (AVG) ameliorates waterlogging-induced damage in cotton by inhibiting ethylene synthesis and sustaining photosynthetic capacity. Plant Growth Regul. 76, 83-98. doi: 10.1007/s10725-015-0037-y

Ray, D. K., Gerber, J. S., Macdonald, G. K., and West, P. C. (2015). Climate variation explains a third of global crop yield variability. Nat. Comm. 6, 1-9. doi: 10.1038/ncomms6989

Simmonds, N. W. (1995). The relation between yield and protein in cereal grain. J. Sci. Food Agric. 67, 309-315. doi: 10.1002/jsfa.2740670306

Ullah, N., and Chenu, K. (2019). "Impact of post-flowering heat stress on staygreen and grain development in wheat," in Proceedings of the Australian Agronomy Conference (Wagga Wagga, NSW), 1-4.

UNICEF (2020). Global Nutrition Report. Available online at: https:// globalnutritionreport.org/reports/2020-global-nutrition-report/ (accessed October 12, 2021).

Wessells, K. R., and Brown, K. H. (2012). Estimating the global prevalence of zinc deficiency: results based on zinc availability in national food supplies and the prevalence of stunting. PLoS ONE 7:e50568. doi: 10.1371/journal.pone. 0050568

Author Disclaimer: All the views expressed in this editorial are authors' own views and do not necessarily reflect the opinion of their respective organizations.

Conflict of Interest: The authors declare that the research was conducted in the absence of any commercial or financial relationships that could be construed as a potential conflict of interest.

Publisher's Note: All claims expressed in this article are solely those of the authors and do not necessarily represent those of their affiliated organizations, or those of the publisher, the editors and the reviewers. Any product that may be evaluated in this article, or claim that may be made by its manufacturer, is not guaranteed or endorsed by the publisher.

Copyright (C) 2022 Ahmad, Ullah, Xu and El Sabagh. This is an open-access article distributed under the terms of the Creative Commons Attribution License (CC BY). The use, distribution or reproduction in other forums is permitted, provided the original author(s) and the copyright owner(s) are credited and that the original publication in this journal is cited, in accordance with accepted academic practice. No use, distribution or reproduction is permitted which does not comply with these terms. 